\title{
Psychometric properties of the Urdu version of the World Health Organization's quality of life questionnaire (WHOQOL-BREF)
}

\author{
Fahad Saqib Lodhi ${ }^{1}$, Owais Raza ${ }^{1}$, Ali Montazeri ${ }^{2}$, Saharnaz Nedjat ${ }^{3}$, Mehdi Yaseri ${ }^{3}$, Kourosh Holakouie-Naieni $^{3 *}$
}

Received: 11 Aug $2016 \quad$ Published: 25 Dec 2017

\begin{abstract}
Background: The present study was conducted to translate and validate the World Health Organization's Quality-of-Life Scale BREF into local language of Pakistan.

Methods: A forward- backward translation procedure was followed to develop the Pakistani version of the questionnaire. Through a multi- stage clustered sampling technique, a sample of individuals aged 18 years and above completed the questionnaire in Abbottabad district, Pakistan. Psychometric properties of the instrument including reliability (internal consistency and test-retest analysis), validity (known group comparison and items' correlation) and their domains were assessed. Satisfactory results were also shown in the correlation matrix in all domains.

Results: A total of 2060 participants were recruited in this study. Participants' mean age was 35.51 (SD=14.31) years in healthy individuals and $39.29(\mathrm{SD}=14.31)$ years in diseased individuals. The internal consistency of the WHOQOL-BREF (Pakistani Version) was 0.86 . Moreover, the physical, psychological, and environmental domains had acceptable reliability (alpha $=0.78,0.75$, and 0.73 , respectively), but reliability was low (alpha $=0.56)$ in the social domain. Reproducibility of the WHOQOL-BREF was as follows: ICC range: $0.72-0.92$ at 2-week retest interval. After performing comparison analysis, the results indicated that the questionnaire significantly segregated the study groups in all QoL domains, except for social relationship.

Conclusion: The study provided strong exploratory evidence for the reliability and validity of the WHOQOL-BREF for use in Pakistan. However, more exploration is needed to improve the reliability results in the social domain.
\end{abstract}

Keywords: Reliability, Quality of life, Validity, Pakistan, WHOQOL-BREF

Copyright $\odot$ Iran University of Medical Sciences

Cite this article as: Saqib Lodhi F, Raza O, Montazeri A, Nedjat S, Yaseri M, Holakouie-Naieni K. Psychometric properties of the Urdu version of the World Health Organization's quality of life questionnaire (WHOQOL-BREF). Med J Islam Repub Iran. 2017 (25 Dec);31:129. https://doi.org/10.14196/mjiri.31.129

\section{Introduction}

Quality of life (QOL) is defined by the World Health Organization Quality of life (WHO-QOL) appraisal group as "individuals perceptions of their position in life in the context of the culture and value systems in which they live and in relation to their goals, expectations, standards, and concerns" (1). WHOQOL-BREF is a short version of WHOQOL-100 developed under the supervision of WHO

Corresponding author: Dr Kourosh Holakouie-Naieni, holakoik@hotmail.com

1. Department of Epidemiology and Biostatistics, School of Public Health, International Campus, Tehran University of Medical Sciences (IC-TUMS), Tehran, Iran.

2. Institute for Health Sciences Research, Academic Center for Education, Culture and Research, Tehran, Iran.

3.Department of Epidemiology and Biostatistics, School of Public Health, Tehran University of Medical Sciences, Tehran, Iran
(2). Different countries such as Japan (3), Iran (4), Croatia (5), India (6), and United Kingdom (7) use WHOQOL. Among several general instruments to measure quality of life, WHOQOL-BREF is one of the most suitable instruments, which is comparatively brief, acceptable to use, valid across cultures, and extensively used in different epi-

$\uparrow$ What is "already known” in this topic:

World Health Organization's quality of life questionnaire (WHOQOL-BREF) scale is the most widely used instrument to measure the quality of life among a different population. However, this is the first study to validate this instrument in the Urdu language to be used among Pakistani urdu speaking population.

$\rightarrow$ What this article adds:

Psychometric properties of Urdu version of the World Health Organization's quality of life questionnaire (WHOQOL-BREF) scale was satisfactory. Thus, as a valid tool, it can be used for measuring quality if life among Pakistani population. 
demiological studies (8). WHOQOL-BREF has been translated and used in many different studies around the world and produced valid psychometric results in New Zealand $(9,10)$, S pain (11), Germany (12), Iran $(13,14)$, Nigeria (15), Taiwan (16), Malawi (17), Brazil (18), and many more countries. To the best of our knowledge, currently there is no version available in Urdu language, which is the official language in Pakistan. Thus, the purpose of this study was to construct a culturally valid Urdu translation of WHOQOL-BREF and assess its reliability and validity. We believed that this questionnaire will be a valuable tool to apply in epidemiological studies. It will also enable us to describe quality of life in Pakistani population and make comparisons across populations.

\section{Methods}

\section{The questionnaire}

WHOQOl-BREF is a 26- item self- administered questionnaire and covers 4 dimensions of QOL ( 6 items for psychological, 7 for the physical, 3 for social, and 8 for environmental domains). The score for each question has response categories ranging from 1 to 5 , with a high score indicating high QOL except in 3 questions targeting pain and discomfort, need for medical treatment, and negative feeling (19). The physical health dimension includes 7 items (mobility, daily life activities, pain, sleep, functional capacity, and energy). The psychological domain assesses negative thinking, self-image, positive approach, self-esteem, mindset, ability to learn, memory, consolidation, religion, and psychic condition. Questions such as social support, sex-life, and personal relationship are categorized in the social relationship domain. Environmental health domain includes questions on financial assets, security, health and social services, living in natural environment, opportunities to achieve advance learning experience, relaxation, and natural environment like air, noise, pollution, and transportation (20). It relatively indicates the subjective response than the objective, by appraisal being made over the previous 2 weeks. Domains' scores were scaled in a positive direction, and the total raw score for these 5 dimensions were transformed into 0 to 100 scale, based on standardized criteria defined by the user manual of WHO-QOL (19). Then, the analysis of this reconstruct score was done, with a higher score in each domain reflecting better quality of life.

\section{Translation}

Translation of the WHO-QOL BREF from English to Urdu (national language of Pakistan) was independently done by a group of experienced bilingual Pakistani health professionals. The translation committee comprising 2 bilingual health professionals and 2 English teachers from a reputed college produced the consolidated forward version. This translated Urdu version was given to another group of health professionals and English teachers who had no previous knowledge of the questionnaire, to back- translate the Urdu version into English. The translation committee made some changes before finalizing the provisional version of the Urdu questionnaire (21). To assess the feasibility and clarity of the items, a pilot study was conducted on 30 individuals conveniently selected from the study area to respond to the questionnaire and also specify those items which were uneasy to understand, complicated, or offensive for them.

\section{Study population, data collection, and study design}

This study was conducted in Abbottabad district with a population of more than 0.8 million (22). This was a population- based cross- sectional study. Multi- stage clustered sampling technique was used and performed in all 52 union councils of Abbottabad district, Khaber Pkutunkhua province, Pakistan, from March 2015 to August 2015.

\section{Recruitment}

In this study, participants were randomly selected from both nuclear and joint families from all 52 union councils of Abbottabad district, Pakistan. The following criteria were used for selection: (1) age 18 years and above (2), mentally stable to answer questions (3), and permanent resident of union councils for at least 5 years. Guests and temporary residents were excluded from the study.

To select the sample from both types of family system, we used multi - stage cluster sampling technique. Abbottabad district consisted of 52 union councils, all of which were included in the study. Each union council was further divided into several more blocks in the shape of Muhalla (Muhalla is a small adjacent area of a union council). We did proportionate sampling according to the 1998 census population (22) of UCs to select Muhalla for the next stage. In the first stage, we randomly selected these blocks (Muhalla) using simple random sampling technique. In the next stage, we selected number of households in that selected block using a random sampling technique again. In each union council, the size of both types of households was proportional to the population size of that union council. Questioners were administered by a trained health worker of each union council through a face to face interview. To ensure confidentiality and discretion, interviews were conducted in a separate room or area detached from other members of the family.

To assess test-retest reliability, every 10th house was selected from each $52 \mathrm{UCs}$, and the same questionnaire was administered on the same household member twice in a 2week interval.

\section{Ethics}

This study was approved by the Ethics Committee of Tehran University of Medical Sciences and by health and political administrative bodies of Abbottabad District, Pakistan. All participants provided verbal and written consent prior the interview.

\section{Statistical analysis}

To assess psychometrical properties of the WHOQOL$\mathrm{BREF}$, different statistical tests were used as follow:

\section{Internal consistency}

Cronbach's $\alpha$ was used to calculate internal consistency for each domain of the WHOQOL-BREF. If the values 
were equal or greater than 0.70 , then they would have been considered as satisfactory (23).

\section{Content validity}

The Urdu version was sent to 6 health experts (4 academic and 2 health experts), and they were requested to assess every item on a 4-point scale ( $1=$ not relevant, $2=$ only relevant if phrasing is strongly adjusted, $3=$ relevant with some adjustment to phrasing, $4=$ very relevant). Experts proposed some suggestions to improve each question. Then, based on the judged relevance per item, the so-called CVI (content validity index) can be calculated (24). CVI is the proportion of experts that judge an item as content valid (a score of 3 or 4). The starting point is a CVI score of at least 0.78 or higher, which should be maintained.

\section{Test-retest}

Intraclass correlation (ICC) is an estimation of the portion of the total measurement variability due to modification among individuals (23). For test - retest, out of 2060 households from all $52 \mathrm{UCs}$, we randomly selected 1 household from each UC and asked them to complete the questionnaire within 2 weeks; these 52 households did not participate in the main study. Reliability of WHOQOLBREF was estimated in a randomly selected sample of individuals performing ICC analysis using SPSS 20 software. Statistical significance was set at 0.05 . For analysis, we expected ICCs of all 4 domains of WHOQOL-BREF to be equal to $0.70(25)$.

\section{Known groups' comparison}

WHOQOL-BREF is able to differentiate disease free individuals from the diseased; this was proved by performing an analysis of covariance (ANCOVA) that controlled confounding effects such as age, sex, education, and marital status, place of residences, occupation, and residence ownership. Factors that independently affected the domain scores and indicated a significant effect in ANCOVA were considered as potential confounders. Significantly high scores in all 4 domains were expected in the disease free individuals when we compared them with those of the diseased group.

\section{Correlation matrix of the WHOQOL-BREF}

The correlation between items and domains was assessed. It was expected that item score would correlate more with the domains to which they were basically accredit. Good items should be sufficiently correlated with others, especially with items in the same domain. Correlation values equal or greater than 0.40 were considered satisfactory, 0.41 to 0.60 good, 0.61 to 0.80 very good, 0.81 to 1.0 as excellent, and 0.21 to 0.40 , and 0.20 as fair and poor, respectively (26).

\section{Results}

\section{Pilot testing}

No obstacle was faced either in item or response section of the questionnaire. Despite all this, minor changes were made based on the results of the pretest. Because Pakistan is a Muslim country, the question regarding sexual life in the questionnaire was changed into "sexual relationship" for the married and "relationship with the opposite sex" for never married, divorced, and separated participants.

A single questionnaire of WHOQOL-BREF takes 4 to 12 minutes to complete (median $=7$ minutes).

\section{The study sample}

Characteristics of the study participants are presented in Table 1 . The total sample size for this study was 2116 . Out of this, 56 households refused to participate in this study, so the analyses were restricted to 2060 participants. Of them, 1600 participants $(77.6 \%)$ had no illness and 460 $(22.4 \%)$ were ill. In this study, illness was taken as a medical status recognized by a physician at least one month before the study. With respect to age distribution, there were

Table 1. Characteristics of the study participants $(n=2060)$

\begin{tabular}{|c|c|c|c|c|}
\hline Characteristics & $\begin{array}{c}\text { All participants } \\
\mathrm{n}=2060\end{array}$ & $\begin{array}{c}\text { Healthy individuals } \\
\mathrm{n}=1600(\%)\end{array}$ & $\begin{array}{c}\text { Diseased- individuals } \\
\mathrm{n}=460(\%)\end{array}$ & $\mathrm{p}$ \\
\hline \multicolumn{5}{|l|}{ Age (years) Mean $\pm \mathrm{SD}$} \\
\hline & $37.89(13.26)$ & $35.51 \pm 11.73$ & $46.12 \pm 14.87$ & $<0.001$ \\
\hline \multicolumn{5}{|l|}{ Education (\%) } \\
\hline No Education & 322 & $227(14.2)$ & $95(20.6)$ & \\
\hline Can read/write & 211 & $159(9.9)$ & $52(11.4)$ & $<0.001$ \\
\hline Madrassa & 45 & $35(2.1)$ & $11(2.4)$ & \\
\hline Primary (up to grade 5) & 636 & $481(30.1)$ & $154(33.5)$ & \\
\hline Secondary (up to grade 12 ) & 658 & $547(34.2)$ & $111(24.1)$ & \\
\hline Tertiary (up to grade 16) & 188 & $151(9.4)$ & $37(8)$ & \\
\hline \multicolumn{5}{|l|}{ Gender } \\
\hline Male & 1055 & $817(51.1)$ & $238(51.7)$ & \\
\hline Female & 1005 & $783(48.9)$ & $222(48.3)$ & 0.700 \\
\hline \multicolumn{5}{|l|}{ Marital status } \\
\hline Married & 1638 & $1256(78.5)$ & $382(83.0)$ & \\
\hline Widow & 60 & $27(1.7)$ & $33(7.1)$ & \\
\hline Divorced & 6 & $4(0.3)$ & $2(0.4)$ & $<0.001$ \\
\hline Separated & 7 & $5(0.3)$ & $2(0.4)$ & \\
\hline Never Married & 349 & $308(19.3)$ & $41(8.9)$ & \\
\hline \multicolumn{5}{|l|}{ Residence place } \\
\hline Urban & 598 & $449(28.1)$ & 149 (32.4) & \\
\hline Rural & 1462 & $1151(71.9)$ & $311(67.6)$ & 0.085 \\
\hline
\end{tabular}




\begin{tabular}{lccc}
\hline Table 1. Cntd & & & \\
\hline Occupation & 810 & $615(38.4)$ & $195(42.4)$ \\
Not working & 78 & $54(3.4)$ & $24(5.2)$ \\
Agriculture & 473 & $379(23.7)$ & $94(20.4)$ \\
Services & 54 & $37(2.3)$ & $17(3.7)$ \\
Domestic worker & 90 & $68(4.3)$ & $22(4.8)$ \\
Retired & 555 & $447(27.9)$ & $108(23.5)$ \\
others & & & $350(76.1)$ \\
Ownership of residence & 1599 & $1249(78.1))$ & $110(23.9)$ \\
Owner & 461 & $351(21.9)$ & 0.296 \\
Not owner & & & \\
\hline
\end{tabular}

$1356(65.82 \%)$ in 18 to 40 years age group, $386(18.7 \%)$ in the 41 to 50 years age group, and 320 in the 50 years and above age group, with a mean age of 37.89 years $(\mathrm{SD}=$ 13.26). There were $1162(56.40 \%)$ employed participants in the study, $90(4.36 \%)$ retired, and $810(39.32 \%)$ unemployed.

There were no statistically significant differences in gender, occupation, place of residence, and ownership of residence between the 2 groups. However, age ( $p \leq 0.001)$, marital status $(\mathrm{p} \leq 0.001)$, and respondent's education $(\mathrm{p} \leq$ 0.001 ) were significantly associated with diseased and nondisease groups. The health conditions mentioned by the participants included hypertension (7.5\%), diabetes $(2.8 \%)$, musculoskeletal pains (8.5\%), physical disabilities (1.4\%), and depression $(1.3 \%)$.

\section{Data quality}

There was no evidence of ceiling or floor effect for any of the items in the data file as responses to all items of questionnaire were fairly distributed across the scale.

\section{Descriptive statistics and internal consistency}

Internal consistency of all the 4 WHOQOL-BREF domains for the total subject population with the mean score, standard deviation, and each subgroup is presented in Table 2. Cronbach's alpha for the total population was 0.86 , which was acceptable for the physical $(0.78)$, psychological (0.71), environmental (0.73), and health domains, but was not acceptable for the social relationship domain (0.56). For this domain, the Cronbach's alpha would have increased to 0.70 if Item 22 (social support) had been deleted. Cronbach's Alpha coefficient was high for the diseased group compared to the disease free group.

\section{Content validity}

Experts rated the relevance of the Urdu Who Qol Bref items and calculated CVI accordingly. The CVI score ranged from 0.78 to 1.00 .

\section{Test- retest}

The interclass correlation for the 4 domains were within the acceptable range after test-retest analysis $(0.71,0.72$, $0.70,0.78$; respectively). ICC for the overall QOL and the general health items was 0.74 .

\section{Known group's comparison}

QoL was compared between the 2 groups to further support discriminant validity of the instrument by adopting known group comparison method (Table 3). As expected, there was a significant difference between the 2 groups in adjusted QoL scores. The diseased group showed significantly low scores in all the 4 domains compared to the disease free group $(\mathrm{p}<0.05)$. In physical domain, confounders were age, gender, education level, and marital status; in psychological domain, they were age, gender, and education level; in social relationship, they were education level and marital status; and in environmental domains, they were gender and education level.

\section{WHOQOL-BREF correlation matrix}

The item scale correlation matrix between each item and all the 4 domains are presented in Table 4 . Out of 24 items that created the domains, 19 were associated with the domain to which they were allocated and met the item scale correlation requirement of $\geq 0.4$. A total of 5 questions, 2 from psychological health domain (bodily image and negative feelings), 1 from social relationship domain (social

Table 2. Descriptive and reliability statistics for the WHOQOL-BREF

\begin{tabular}{|c|c|c|c|c|c|}
\hline \multirow[b]{2}{*}{ Domains } & \multicolumn{2}{|r|}{ Descriptive statistics } & \multicolumn{2}{|c|}{ Alpha coefficients } & \multirow[b]{2}{*}{$\begin{array}{c}\text { Diseased- Individuals } \\
(\mathrm{n}=460)\end{array}$} \\
\hline & $\begin{array}{l}\text { No. of } \\
\text { items }\end{array}$ & $\begin{array}{c}\text { ALL }(\mathrm{N}=2060) \\
\text { Mean score }(\mathrm{SD})\end{array}$ & $\begin{array}{c}\text { All } \\
(\mathrm{n}=2060)\end{array}$ & $\begin{array}{l}\text { Healthy individuals } \\
(\mathrm{n}=1600)\end{array}$ & \\
\hline Physical health & 7 & $25.18(4.26)$ & 0.78 & 0.75 & 0.80 \\
\hline Psychological health & 6 & $22.18(3.56)$ & 0,71 & 0.70 & 0.73 \\
\hline Social relationships & 3 & $11.63(1.97)$ & 0.56 & 0.56 & 0.53 \\
\hline Environmental health & 8 & $22.75(4.72)$ & 0.73 & 0.72 & 0.74 \\
\hline
\end{tabular}

Table 3. The known groups' comparison (controlled for confounders)

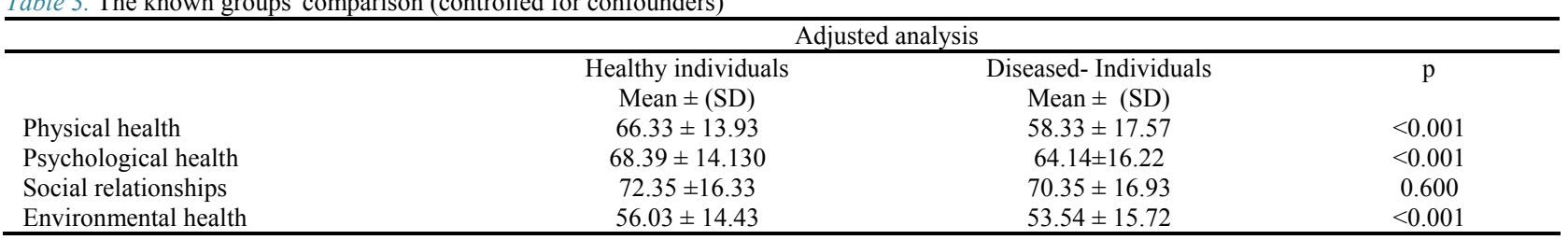


Table 4. Item-scale correlation matrix for the four WHOQOL-BREF measures ( $\mathrm{n}=2060)$

\begin{tabular}{|c|c|c|c|c|}
\hline & Physical health & Psychological health & Social health & Environmental health \\
\hline \multicolumn{5}{|l|}{ Physical Health (Item number) } \\
\hline Pain (3) & 0.43 & 0.24 & 0.11 & 0.13 \\
\hline Dependence of medical aids (4) & 0.45 & 0.29 & 0.10 & 0.12 \\
\hline Energy (10) & 0.56 & 0.53 & 0.26 & 0.42 \\
\hline Mobility (15) & 0.65 & 0.44 & 0.23 & 0.36 \\
\hline Sleep and rest (16) & 0.44 & 0.40 & 0.29 & 0.33 \\
\hline Activities of daily living (17) & 0.61 & 0.47 & 0.29 & 0.36 \\
\hline Work capacity (18) & 0.59 & 0.48 & 0.31 & 0.36 \\
\hline \multicolumn{5}{|l|}{ Psychological health (Item number) } \\
\hline Positive feeling (5) & 0.35 & 0.55 & 0.34 & 0.44 \\
\hline Personal belief (6) & 0.34 & 0.53 & 0.26 & 0.37 \\
\hline Concentration (7) & 0.51 & 0.47 & 0.26 & 0.36 \\
\hline Bodily image (11) & 0.35 & 0.38 & 0.29 & 0.31 \\
\hline Self-esteem (19) & 0.54 & 0.47 & 0.42 & 0.37 \\
\hline Negative feeling (26) & 0.26 & 0.27 & 0.16 & 0.25 \\
\hline \multicolumn{5}{|l|}{ Social relationships (Item number) } \\
\hline Personal relationship (20) & 0.30 & 0.41 & 0.47 & 0.29 \\
\hline Sexual activity (21) & 0.24 & 0.37 & 0.47 & 0.28 \\
\hline Social support (22) & 0.21 & 0.22 & 0.22 & 0.35 \\
\hline \multicolumn{5}{|l|}{ Environmental health (Item number) } \\
\hline Security (8) & 0.35 & 0.46 & 0.29 & 0.38 \\
\hline Physical environment (9) & 0.15 & 0.18 & 0.18 & 0.28 \\
\hline Financial support (12) & 0.31 & 0.47 & 0.30 & 0.48 \\
\hline Accessibility of information (13) & 0.32 & 0.41 & 0.26 & 0.46 \\
\hline Leisure activity (14) & 0.25 & 0.27 & 0.22 & 0.40 \\
\hline Home environment (23) & 0.24 & 0.26 & 0.31 & 0.40 \\
\hline Health care $(24)$ & 0.23 & 0.25 & 0.24 & 0.67 \\
\hline Transport (25) & 0.22 & 0.30 & 0.22 & 0.50 \\
\hline
\end{tabular}

support), and 2 from environmental health domain (security and physical environmental) did not meet this requirement. Within each of the 4 domains, items are significantly correlated at 0.01 level.

\section{Discussion}

The present study tested the psychometric properties of the newly developed Urdu version of WHOQOL-Bref in 2 groups selected from the general population of Pakistan. The study included a comparatively large representative sample from the general population. The sample was collected both from urban and rural population; most of the participants were illiterate and lived in the rural area, so to avoid selection bias and reduce missing data, we used interviews. Based on the promising study results, the WHOQOL-BREF instrument can be used to assess quality of life in various populations in Pakistan.

Sociodemographic characteristics, such as mean age, marital status, and education, were found to be significantly associated with disease and non-disease groups $(\mathrm{p}<0.001)$ (Table 1). As the Pakistanis grow older, they will be more prone to such diseases as hypertension, diabetes, anxiety, depression, and ischemic heart diseases $(27,28)$. Similarly, in Pakistani population, education and marital status also play a vital role to live a healthy life (29).

Content validity of WHOQOL-BREF was analyzed by an experienced panel and found to be adequately secured. Kappa statistic was above 0.75 for all items, and according to previous parameters (30), it indicated excellent and high agreement. Values of CVI indicated acceptable culture relevance (31) and 0.75 was the least recommended value for an item, indicating good content validity.

Discriminant validity was assessed and confirmed by known group comparison, and there was a significant difference between the 2 groups after controlling for age, sex, education, marital status, place of residence, occupation, and residence ownership in the physical, psychological, and environmental health domains, but not in the social relationship domain. In another study, environment domain showed non-significant difference between the 2 groups (32). A study conducted by Skevington et al. in 2004 showed good discriminant validity in all 4 domains. After exploring the outcome thoroughly, we found that multiple filed trial centers like UK, Spain, Brazil, Norway, Italy (8) etc. failed to differentiate between healthy and diseased respondents, particularly in social relationship and environment domains. In this study, data analysis confirmed that the non-diseased group enjoyed better quality of life compared to the diseased group (Table 3). Differentiation was highest in the physical and psychological domains, followed by the environmental domain. This finding is an implication of the uniqueness of the WHOQOL-BREF to clearly separate the disease and non-disease groups.

The questionnaire's reproducibility was measured using intraclass correlation, ICC of the tool was $>0.80$, producing good test-retest reliability of the scale $(24,25)$. The study results indicated satisfactory alpha coefficients in all domains of the WHOQOL-BREF, except for the social relationship. Results of other validation studies have shown similar results, reporting Cronbach's alpha coefficient of less than 0.7 in the social relationship domain $(12,14,26$ 28). The small Cronbach's alpha coefficient was probably due to the fact that the social relationship domain was made up of only 3 items; moreover, interpersonal relationships and sexual activities are comparatively different notions in Pakistan's culture. Furthermore, female communication within the society is confined in Pakistan. 
Item-scale correlation matrix for the WHOQOL-BREF measures showed that all items of physical, psychological, relationship, and environmental health domains had a significant correlation with their respective domains. Questions with week correlation were negative feelings from psychological domain, social support from social relationship domain, and physical environmental from environmental domain. The result is somewhat different from those reported in Iran by Nedjat (13) and Ghassemi (14), who showed that the validity item has higher correlation coefficients with their respective health domains. This difference might have been due to cultural variation among people or translation problems (6).

Our study revealed that WHOQOL BREF has good reliability and validity. Similar types of results were shown in multiple quality of life studies including assessment of quality of life in Taiwan (33), people with dementia (34), Greek population (35), Spanish patients with other health conditions such as schizophrenia (11), Koreans with physical impairment (36), Iranian adult (14), and Iranian population with physical and psychological health (13).

There were also several limitations in this study, which need to be considered. Our study focused on verbal responses against the probing queries from the data collector. Because many interviewers were part of the study, there might have been a difference in the probing methods, influencing participant's response. However, it was controlled by providing proper training to all interrogators, consulting with regional cultural mentors, and forming the procedures for proper interviews and investigations. The same questionnaire was used for urban and rural population, and this might have caused some missing data in both groups. This needs to be further explored. Another limitation of this study was not using a similar age range for both groups involved. Furthermore, we did not apply factor analysis in our study. Further studies should be conducted to consider the limitation of the present study.

\section{Conclusion}

The study provided strong exploratory evidence for the reliability and validity of the WHOQOL-BREF for use in Pakistan. However, more exploration is needed to improve the reliability results in the social domain.

\section{Acknowledgements}

This study was a part of $\mathrm{PhD}$ dissertation in Epidemiology. We thank all those who have helped us in conducting this study. Our thanks go to all participants, the health workers of Abbottabad district for their help in data collection process from all union councils of the district. We also extend our gratitude to Dr. Umer Farooq, Dr. Faisal Khanzada and Dr. Qasim for their permission and kind supervision in the project. Bilingual panel: Dr. Ashfaq, Sulail Fatima, Syed Assad Shah, and Dr. Saadia irum. The authors had full control over all primary data.

\section{Conflict of Interests}

The authors declare that they have no competing interests.

References

1. Groupt W. Study protocol for the World Health Organization project to develop a Quality of Life assessment instrument (WHOQOL). Qual Life Res. 1993;2(2):153-9.

2. Skevington SM, Sartorius N, Amir M. Developing methods for assessing quality of life in different cultural settings. Soc Psychiatry Psychiatr Epidemiol. 2004;39(1):1-8.

3. Tazaki M, Nakane Y, Endo T, Kakikawa F, Kano K, Kawano H, et al. Results of a qualitative and field study using the WHOQOL instrument for cancer patients. Jpn J Clin Oncol. 1998;28(2):134-41.

4. Zayeri F. Psychometric properties of the Persian version of the World Health Organization's quality of life questionnaire (WHOQOL-100). Arch Iran Med. 2011;14(4):281.

5. Pibernik-Okanović M, Szabo S, Metelko Ž. Quality of life following a change in therapy for diabetes mellitus. Pharmacoeconomics. 1998;14(2):201-7.

6. Dandona R, Dandona L, McCarty CA, Rao GN. Adaptation of WHOQOL as health-related quality of life instrument to develop a vision-specific instrument. Indian J Ophthalmol. 2000;48(1):65.

7. Skevington SM, Carse MS, Williams ACdC. Validation of the WHOQOL-100: pain management improves quality of life for chronic pain patients. Clin J Pain. 2001;17(3):264-75.

8. Skevington SM, Lotfy M, O'Connell KA. The World Health Organization's WHOQOL-BREF quality of life assessment: psychometric properties and results of the international field trial. A report from the WHOQOL group. Qual Life Res. 2004;13(2):299-310.

9. Krägeloh CU, Kersten P, Billington DR, Hsu PHC, Shepherd D, Landon J, et al. Validation of the WHOQOL-BREF quality of life questionnaire for general use in New Zealand: Confirmatory factor analysis and Rasch analysis. Qual Life Res. 2013;22(6):1451-7.

10. Krägeloh C, Henning M, Hawken S, Zhao Y, Shepherd D, Billington R. Validation of the WHOQOL-BREF quality of life questionnaire for use with medical students. Education for health. 2011;24(2):545.

11. Lucas-Carrasco R. The WHO quality of life (WHOQOL) questionnaire: Spanish development and validation studies. Qual Life Res. 2012;21(1):161-5.

12. Trompenaars FJ, Masthoff ED, Van Heck GL, Hodiamont PP, De Vries J. Content validity, construct validity, and reliability of the WHOQOL-Bref in a population of Dutch adult psychiatric outpatients. Qual Life Res. 2005;14(1):151-60.

13. Nedjat S, Montazeri A, Holakouie K, Mohammad K, Majdzadeh R. Psychometric properties of the Iranian interview-administered version of the World Health Organization's Quality of Life Questionnaire (WHOQOL-BREF): a population-based study. BMC Health Serv Res. 2008;8(1):61.

14. Usefy A, Ghassemi GR, Sarrafzadegan N, Mallik S, Baghaei A, Rabiei K. Psychometric properties of the WHOQOL-BREF in an Iranian adult sample. Community Ment Health J. 2010;46(2):139-47.

15. Akinpelu A, Maruf F, Adegoke B. Validation of a Yoruba translation of the World Health Organization's quality of life scale--short form among stroke survivors in Southwest Nigeria. Afr J Med Med Sci. 2006;35(4):417-24.

16. Chang KC, Wang JD, Tang HP, Cheng CM, Lin CY. Psychometric evaluation, using Rasch analysis, of the WHOQOL-BREF in heroindependent people undergoing methadone maintenance treatment: further item validation. Health Qual life outcomes. 2014;12(1):148.

17. Colbourn T, Masache G, Skordis-Worrall J. Development, reliability and validity of the Chichewa WHOQOL-BREF in adults in lilongwe, Malawi. BMC Res notes. 2012;5(1):346.

18. Berlim MT, Pavanello DP, Caldieraro MA, Fleck MP. Reliability and validity of the WHOQOL BREF in a sample of Brazilian outpatients with major depression. Qual Life Res. 2005;14(2):561-4.

19. Group TW. The World Health Organization quality of life assessment (WHOQOL): development and general psychometric properties. Soc Sci Med. 1998;46(12):1569-85.

20. Whoqol W. WHOQOL-Brief introduction, administration, scoring and generic version of the assessment. WHO December. 1996.

21. Sperber AD. Translation and validation of study instruments for cross-cultural research. Gastroenterology. 2004;126:S124-S8.

22. Haq M, Mustafa UU, Ahmad I. Household's willingness to pay for safe drinking water: A case study of Abbottabad district. Pakistan Development Review. 2007;46(4 Part II):1137-53.

23. Mykletun A, Stordal E, Dahl AA. Hospital Anxiety and Depression (HAD) scale: factor structure, item analyses and internal consistency in a large population. Br J Psychiatry. 2001;179(6):540-4.

24. Lynn MR. Determination and quantification of content validity. Nurs Res. 1986;35(6):382-6. 
25. Bonomi AE, Patrick DL, Bushnell DM, Martin M. Validation of the United States' version of the World Health Organization Quality of Life (WHOQOL) instrument. J Clin Epidemiol. 2000;53(1):1-12.

26. Bernstein IH, Nunnally J. Psychometric theory. New York: McGrawHill Oliva, TA, Oliver, RL, \& MacMillan, IC (1992) A catastrophe model for developing service satisfaction strategies. Journal of Marketing. 1994;56:83-95

27. Aziz K, Faruqui A, Teri M, Davis C, Abenathy J. Blood pressure and hypertension distribution in a lower middle class urban community in Pakistan. J Pak Med Assoc.2005;55(8):333-8.

28. Khuwaja AK, Lalani S, Dhanani R, Azam IS, Rafique G, White F. Anxiety and depression among outpatients with type 2 diabetes: A multi-centre study of prevalence and associated factors. Diabetol Metab Syndr. 2010;2(1):72.

29. Asghar Z, Attique N, Urooj A, Mahmood N. Measuring impact of education and socio-economic factors on Health for Pakistan. Pak Dev Rev. 2009;48(4):653-74.

30. Cicchetti DV. On a model for assessing the security of infantile attachment: Issues of observer reliability and validity. J Behav Brain Sci. 1984;7(1):149-50.

31. Polit DF, Beck CT, Owen SV. Is the CVI an acceptable indicator of content validity? Appraisal and recommendations. Res Nurs Health. 2007;30(4):459-67.

32. Xia P, Li N, Hau KT, Liu C, Lu Y. Quality of life of Chinese urban community residents: a psychometric study of the mainland Chinese version of the WHOQOL-BREF. BMC Med Res Methodol. 2012;12(1):37.

33. Jang Y, Hsieh $\mathrm{CL}$, Wang $\mathrm{YH}, \mathrm{Wu} \mathrm{YH}$. A validity study of the WHOQOL-BREF assessment in persons with traumatic spinal cord injury. Arch Phys Med Rehabil. 2004;85(11):1890-5.

34. Lucas-Carrasco R, Skevington SM, Gómez-Benito J, Rejas J, March J. Using the WHOQOL-BREF in persons with dementia: a validation study. Alzheimer Dis Assoc Disord. 2011;25(4):345-51.

35. Ginieri-Coccossis M, Triantafillou E, Tomaras V, Soldatos C, Mavreas V, Christodoulou G. Psychometric properties of WHOQOLBREF in clinical and health Greek populations: incorporating new culture-relevant items. Psychiatriki . 2011;23(2):130-42.

36. Min SK, Kim K, Lee C, Jung YC, Suh SY, Kim DK. Development of the Korean versions of WHO Quality of Life scale and WHOQOLBREF. Qual Life Res. 2002;11(6):593-600. 